\title{
TEMPERATURE DEPENDENCE OF MAGNETIZATION IN TRANSITION METAL ULTRATHIN FILMS OF VARIOUS THICKNESSES*
}

\author{
R. ŚWIRKOWICZ \\ Institute of Physics, Warsaw Technical University \\ Koszykowa 75, 00-662 Warszawa, Poland \\ (Received May 6, 1991; in final form July 26, 1991)
}

\begin{abstract}
Temperature dependence of the local magnetization in the spin-wave regime is calculated within the framework of the multiband model for ultrathin films consisting of 5, 7 and 9 monolayers. The temperature range in which the calculated results can be fitted to the Bloch $T^{3 / 2}$ law is found in all cases. The Bloch coefficient $B_{\mathrm{av}}$ corresponding to temperature dependence of the average film magnetization is found to be proportional to $1 / D$, where $D$ is the thickness of the film. The spatial distribution of the local magnetization is obtained. The Bloch coefficient corresponding to the surface layer appears to be greater than the one corresponding to the central layer, namely $B_{5}>B_{\mathrm{c}}$. The ratio $B_{\mathrm{s}} / B_{\mathrm{c}}$ is increasing with an increase of the film thickness. The calculated results are well consistent with experimental ones obtained for ultrathin films of various thicknesses.
\end{abstract}

PACS numbers: $75.70 . A k ; 76.80 .+y$

\section{Introduction}

Temperature dependence of magnetization in ferromagnetic thin films has been investigated theoretically [1-3] as well as experimentally (see e.g. [4-8]). Recently, experiments on ultrathin films consisting of few monolayers have been performed. The Mössbauer method has been used for Fe ultrathin films deposited on $\mathrm{W}$ and $\mathrm{Ag}$ [5-8]. It has been stated that within the spin-wave regime the magnetization of the film decreases according to the Bloch $T^{3 / 2}$ law, namely:

$$
m(T)=m(0)\left(1-B T^{3 / 2}\right) .
$$

\footnotetext{
*Supported by the Project CPBP 01.08 .
} 
Results which fit well to the above law have been obtained for films consisting of more than 20 monolayers (ML) as well as for extremely thin films consisting of 1 or 3 ML [5-8]. The coefficient $B$ appears to be different for surface and central layer magnetization. According to $[6,7]$ the ratio $B_{\mathrm{s}} / B_{\mathrm{c}}$ is greater than 1 and it increases with an increase of the film thickness. The saturation of $B_{\mathrm{s}} / B_{\mathrm{c}}$ is achieved for a film of about 20 ML. For such films the surface magnetization decreases twice as fast as the central layer magnetization. The result $B_{s}=2 B_{\mathrm{b}}$, where $B_{\mathrm{b}}$ corresponds to the bulk magnetization, is characteristic for semi-infinite systems. A temperature dependence of the type (1) with $B_{\mathrm{s}}=2 B_{\mathrm{b}}$ was predicted by Rado [9]. It was also confirmed by microscopic calculations of Mills and Maradudin [10] and of Mathon and Ahmad [11].

On the other hand, calculations performed for very thin films within the framework of the localized electron model lead to the linear dependence of the magnetization in the spin-wave regime $[1,2]$. Though there are some experiments which confirm this result (see [4] for $\mathrm{Co}(111)$ and $48 \mathrm{Ni} / 52 \mathrm{Fe}(111)$ films); the theory presented in [1,2] seems to be inadequate to explain the temperature dependence of magnetization in ultrathin Fe films. The only results obtained for the localized electron model which seem to be consistent with experimental ones are these of Haubenreisser et al. [3]. The method of Green function was used in this case.

Ilowever, up to now, there is no general theory which would allow one to explain all results of measurements for the temperature dependence of magnetization in ultrathin films. The lack of theoretical investigations can be especially seen in the case of the approach based on the itinerant electron model. The first approach to the problem has been presented very recently for films consisting of 7 atomic layers [12]. Consistency of calculations [12] and of measurements performed with the use of Mössbauer spectroscopy seems to be quite good. This encourages us to undertake the deeper and more extensive investigations.

In the present paper the temperature dependence of magnetization within the spin-wave regime is evaluated and profoundly discussed for ultrathin films of various thicknesses. It is pointed out that the most characteristic features reported experimentally for the temperature dependence of magnetization in ultrathin films can be obtained within the framework of the presented approach.

\section{The method of calculation}

The deviation of magnetization in layer $\nu, \Delta m_{\nu}(T)$, is determined as a difference of the magnetization calculated at $T=0 \mathrm{~K}$ and the magnetization at temperature $T$, namely:

$$
\Delta m_{\nu}(T)=m_{\nu}(0)-m_{\nu}(T) .
$$

In the spin-wave regime the deviation $\Delta m_{\nu}(T)$ is given by [11]:

$$
\Delta m_{\nu}(T)=\int_{0}^{\infty} N_{\nu}(E)[\exp (E / k T)-1]^{-1} \mathrm{~d} E .
$$

$N_{\nu}(E)$ denotes here the spin-wave density of states in the layer $\nu$. It can be expressed in terms of imaginary part of the dynamic susceptibility $\chi_{\nu \nu}(q, E)$ in a 
following way:

$$
N_{\nu}(E)=\frac{1}{\pi} \operatorname{Tr} \operatorname{Im} \chi_{\nu \nu}(q, E) .
$$

The mixed Bloch-Wannier representation is used in the above formula and the susceptibility matrix is dependent on a wave vector $q$ parallel to the surface. The trace is taken over the values of the wave vector $q$ from the two-dimensional Brillouin zone. One can see that for calculation of $\Delta m_{\nu}(T)$ the transverse dynamic susceptibility should be determined for various values of $q$ and $E$.

In general, with $\chi(q, E)$ determined a number of quantities characteristic for dynamics of spin systems can be evaluated. Spin-wave energies and dispersion relation can be calculated form the poles of $\operatorname{Im} \chi$. Diagonalization of the matrix Re $\chi$ in the region of very low energies allows one to determine the amplitudes of spin-wave modes in the subsequent atomic layers [13].

The thin film dynamic susceptibility is calculated within the framework of the random phase approximation according to the following formula [14]:

$$
\chi(q, E)=\left[I-\chi^{0}(q, E) U\right]^{-1} \chi^{0}(q, E) .
$$

$\chi^{0}$ denotes here a free-electron susceptibility. Its elements depend strongly on the one-electron Hartree-Fock energies and on the coefficients of expansion of the one-electron wave functions [14]. $U$ corresponds to the effective intraatomic interactions between electrons.

In Ref. [15] the dynamic susceptibility $\chi(q, E)$ is calculated according to formula (5). The multiband approach is used with an assumption that the effective Coulomb and exchange integrals corresponding to the same and different orbital states are simply equal to $U$. Evaluations are performed for nickel ultrathin films consisting of 3, 5, 7 and 9 monolayers (ML). Results obtained in Ref. [15] clearly show that in all films considered acoustic surface mode appears. The energy of the mode practically does not depend on the film thickness (except 3 ML case). For very low $q$ dispersion relation of the surface mode is close to a quadratic one. But it strongly deviates from $D q^{2}$ dependence for slightly greater values of the wave vector. For higher modes, the bulk ones, the energy gap increases strongly with a decrease of the film thickness. Density of states of spin-wave modes is also determined in [15]. Here, it should be mentioned that the surface density of states is considerably enhanced as compared to the central one. The enhancement is obtained in all films considered and it is a result of the presence of acoustic surface modes.

In the present paper the deviation of magnetization in the layer $\nu, \Delta m_{\nu}(T)$, is calculated according to formula (3). The spin-wave density of states $N_{\nu}(E)$ determined in [15] is used. Because of quasi-two-dimensional behavior of density of states in the low energy region (see [15]) divergence at $E=0$ will appear in integral (3). To avoid this, similarly as in Ref. [12], we introduce some effective field simulating the anisotropy effects. The anisotropy energy required for removing the divergence is of the order of the numerical computation error. Therefore, the influence of the anisotropy field on the band structure and spin-wave density of states at slightly higher energies can be practically neglected (see [12]). 


\section{Results}

The temperature dependence of magnetization in subsequent atomic layers is calculated for ultrathin films consisting of 5,7 and $9 \mathrm{ML}$. In all cases considered the surface magnetization decreases with temperature faster than the magnetization of the central layer. In order to illustrate this result the normalized magnetization of surface and central planes is depicted in Fig. 1. According to Fig. 1 it can also be

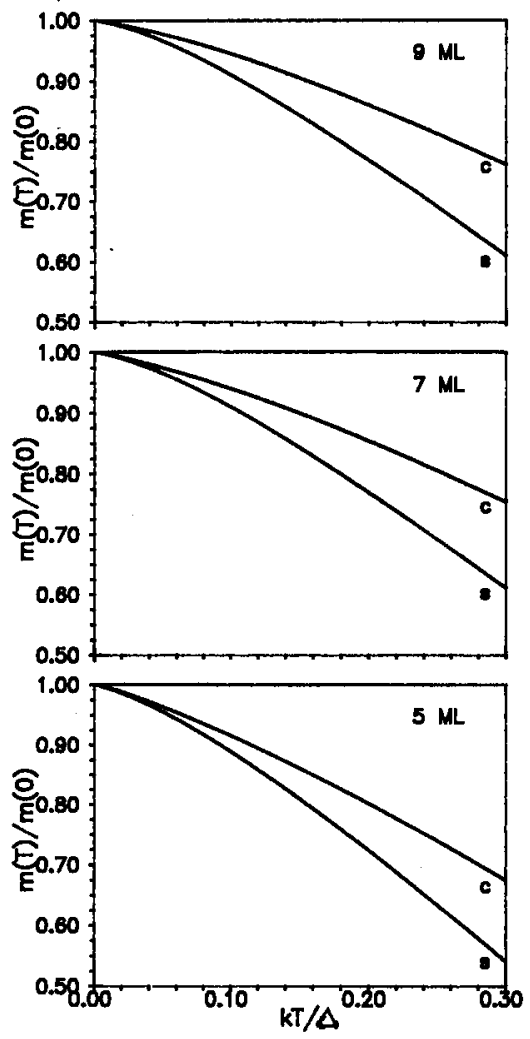

Fig. 1. Temperature dependence of the normalized magnetization of surface (s) and central (c) layers for films consisting of 5, 7, and 9 monolayers (ML).

seen that at given temperature the surface magnetization for $5 \mathrm{ML}$ case is clearly lower than for $9 \mathrm{ML}$ film. The same relation is found for the magnetization of the central layer. Therefore, temperature changes of magnetization appear to be stronger in the case of thinner films.

The normalized mean magnetization of the film in dependence on temperature is depicted in Fig. 2. The curves for films of various thicknesses are presented. The lowest curve in Fig. 2 corresponds to $5 \mathrm{ML}$ film, whereas the upper one to $9 \mathrm{ML}$ film. One can see that the decrease of magnetization is relatively fast in the case of $5 \mathrm{ML}$ film. For thicker films temperature changes of magnetization 


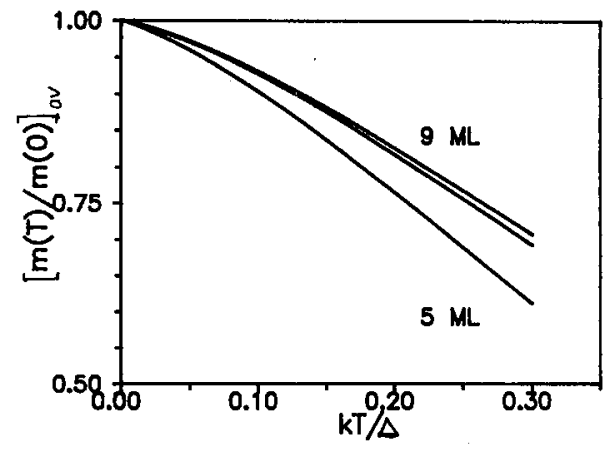

Fig. 2. Temperature dependence of the normalized average film magnetization for films of various thicknesses.

appear to be not so strong: The most gradual decrease of the mean magnetization is obtained for the film consisting of $9 \mathrm{ML}$.

In ultrathin films the magnetization appears to be a complex function of temperature. In the region of very low temperatures (i.e. for $k T / \Delta \approx 0.04$, where $\Delta=0.6 \mathrm{eV}$ denotes the spin-splitting energy) the dependence is close to linear. It is a consequence of the quasi-two-dimensional behavior of spin-wave density of states for low energies (see [15]). However, in the more wide temperature region the calculated magnetization can be fitted to the Bloch $T^{3 / 2}$ law. In Fig. 3 the normalized magnetization of the surface and central layers in dependence on $(k T / \Delta)^{3 / 2}$ is depicted for films of various thicknesses. The dashed line in all cases corresponds to the calculated results, whereas the solid line presents fitting to $T^{3 / 2}$ law. According to Fig. 3 the fitting to the Bloch dependence appears to be quite good up to the temperatures of the order of $k T / \Delta \approx 0.25$. Deviations from the $T^{3 / 2}$ dependence are rather small, especially for thicker films (9 ML case).

Fitting of the local magnetization to the Bloch law allows one to determine the Bloch coefficients $B$ for subsequent atomic layers. The spatial distribution of the parameter $B$ is presented in Fig. 4 for films of various thicknesses. It can be seen that for a film of a given thickness, the coefficient $B$ increases monotonically from central to surface layer, i.e. $B_{\mathrm{s}}$ is greater than $B_{\mathrm{c}}$ in all cases. Values of $B_{\mathrm{s}}$ calculated for 7 and $9 \mathrm{ML}$ films are close to each other, whereas $B_{\mathrm{c}}$ is slightly lower for $9 \mathrm{ML}$ case. However, in $5 \mathrm{ML}$ film the spin-wave parameters $B_{\mathbf{s}}$ and $B_{\mathrm{c}}$ are clearly enhanced as compared to corresponding coefficients calculated for 7 and $9 \mathrm{ML}$ cases. Therefore the Bloch coefficients are dependent on the film thickness. This dependence is more pronounced for thinner films. The ratio $B_{\mathrm{s}} / B_{\mathrm{c}}$ is lower than two for all cases considered. Namely, the values of $B_{\mathrm{s}} / B_{\mathrm{c}}$ equal to $1.43,1.61$ and 1.66 are obtained for $5 \mathrm{ML}, 7 \mathrm{ML}$ and $9 \mathrm{ML}$ films, respectively. One can see that $B_{\mathrm{s}} / B_{\mathrm{c}}$ increases with an increase of the film thickness.

On the other hand, while fitting the mean magnetization of the film to the Bloch dependence it is possible to determine the coefficient $B_{\mathrm{av}}$. The obtained $B_{\mathrm{av}}$ is greater than $B_{\mathrm{c}}$ in all cases considered. Moreover, it appears that the value of 


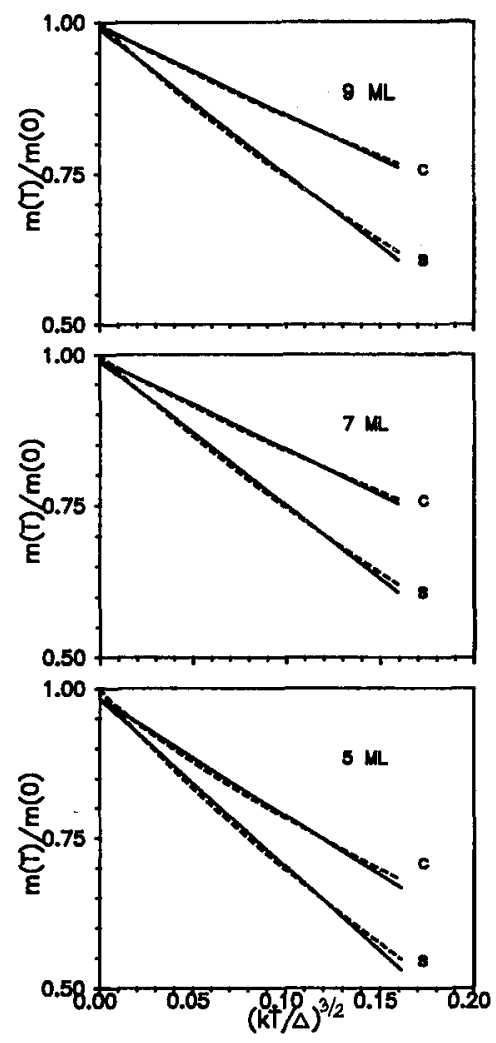

Fig. 3. The normalized magnetization of surface (s) and central (c) layers in dependence on $T^{3 / 2}$.

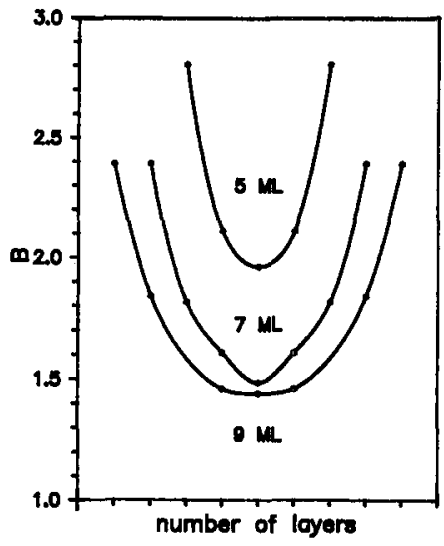

Fig. 4. The spatial distribution of the Bloch coefficient $B$ for films of various thicknesses. 


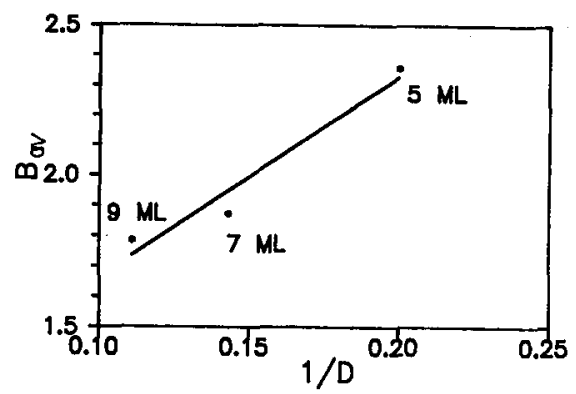

Fig. 5. The average spin-wave parameter $B_{\mathrm{av}}$ as a function of the reciprocal of the film thickness ( $D$ in atomic layers).

$B_{\text {av }}$ decreases with an increase of the film thickness. In Fig. $5 B_{\mathrm{av}}$ is presented in dependence on $1 / D$, where $D$ denotes the thickness of the film. In our case $D$ is equal to 5,7 and 9 for $5 \mathrm{ML}, 7 \mathrm{ML}$ and $9 \mathrm{ML}$ films, respectively. Though only few points are presented in the figure it can be seen that the calculated results relatively well fit to a straight line. Therefore, it seems very likely that $B_{\mathrm{av}}$ is proportional to $1 / D$. However, calculations for other cases are necessary to confirm the conclusion.

\section{Concluding remarks}

The basic result obtained in the paper that the surface magnetization decreases in dependence on temperature faster than the magnetization inside the film is fully confirmed by other theoretical $[10,12]$ and experimental investigations $[5-8,16]$. It should be emphasized that predicted by Rado faster decrease of the surface magnetization is characteristic for semi-infinite systems as well as for ultrathin films.

Within the framework of localized electron model the linear dependence of magnetization is obtained in ultrathin films when spin-wave approximation is used $[1,2]$. This type of dependence is also obtained in nickel ultrathin films considered in the paper, but in definite temperature regions only. In general, the temperature dependence of magnetization appears to be a more complex function. A relatively wide temperature region in which the calculated results can be quite well fitted to the Bloch $T^{3 / 2}$ law is found.

According to Rado [17] the dependence of $m$ on temperature $T$ can be quasi-linear rather than proportional to $T^{3 / 2}$ when the surface anisotropy of a special kind is taken into account. However, at present it seems very difficult to include anisotropy effects within the framework of the approach presented in the paper.

A direct comparison of the results obtained in the paper with the experimental ones still seems to be difficult. The measurements of the magnetization in ultrathin films were performed for $48 \mathrm{Ni} / 52 \mathrm{Fe}(111)$ samples [4]. The results seem to confirm the linear dependence. Considerably thick nickel films at rather high 
temperatures $\left(T>0.5 T_{\mathrm{c}}\right)$ were also investigated [17-20]. Very recently, the temperature dependence of the hyperfine field in ultrathin Ni films deposited on $\mathrm{Cu}(100)$ substrates has been measured [21]. According to obtained results the hyperfine field is a nonlinear function of temperature. However, more detailed experimental data are required for comparison with our theoretical results. On the other hand, it can be seen that the results obtained in the paper for nickel case seem to be quite consistent with the experimental ones for Fe ultrathin films [6, 7]. First of all, the experimental and theoretical results can be quite well fitted to the Bloch law. Though, in nickel case the fitting is good in a definite temperature region only. In both, experimental and theoretical approaches the Bloch coefficient $B_{\mathbf{s}}$ greater than $B_{\mathrm{c}}$ is obtained. Moreover, the ratio $B_{\mathrm{s}} / B_{\mathrm{c}}$ is increasing with an increase of the film thickness. The effect of saturation $B_{\mathrm{s}} / B_{\mathrm{c}}=2$ is not obtained in the paper. The investigated films are simply too thin. However, the value of spin-wave parameter $B_{\mathrm{c}}$ is clearly decreasing with an increase of the film thickness (see Fig. 4). It does mean that in thicker samples the decrease of the central layer magnetization is slower. One can expect therefore that for sufficiently thick films the coefficient $B_{\mathrm{c}}$ will be the smallest and the temperature dependence of the central layer magnetization will appear close to the dependence characteristic for the bulk systems. In such a case the saturation of $B_{\mathrm{s}} / B_{\mathrm{c}}$ will be achieved. To confirm this conclusion fully, calculations performed for considerably thick films are necessary. Computations for films consisting of over $20 \mathrm{ML}$ (as it is suggested by experimental measurements) could be very time-consuming. At present it is rather difficult for us to undertake them.

The fact that the coefficient $B_{\mathrm{av}}$ is decreasing with an increase of the film thickness is also consistent with experimental results. According to Mössbauer measurements for $\mathrm{Fe}$ ultrathin films $B_{\mathrm{av}} \approx 1 / D$ is obtained $[6,7]$. The results presented in the paper for nickel case seem to confirm this relation, though only three points are considered.

However, in general $B_{\text {av }}$ may not be proportional to $1 / D$ (see [22]). Variation of $B_{\mathrm{av}}$ with film thickness can depend on material and on sample preparations. It was found that for polycrystalline $\mathrm{Ni}$ on $\mathrm{Cu}$ substrate $B_{\mathrm{av}}$ is not a linear function of $1 / D$. It should be pointed out that near $\mathrm{Ni}-\mathrm{Cu}$ interface the ground-state magnetization is changed essentially [23]. Such changes influence the spin-wave amplitudes and the coefficient $B$ considerably.

One can see that a qualitative agreement of the results obtained in the paper with the experimental measurements is quite satisfactory. Therefore it seems that the characteristic features of the temperature dependence of magnetization in ultrathin films of transition metals like $\mathrm{Fe}$ and $\mathrm{Ni}$ appear to be the same, at least in a certain temperature region. In Fe as well as in Ni ultrathin films the surface appears to be magnetically stronger and a moderate enhancement of the surface magnetization is obtained. On the other hand, in a case of very strong surface perturbation a great enhancement of the surface magnetization can be observed. The amplitudes of spin-wave modes with lowest energies appear also to be very high in the surface layer. The enhancement of the amplitudes leads to considerable enhancement of the surface density of spin-wave states. Therefore, in such a case faster decrease of magnetization can be expected. 


\section{References}

[1] W. Döring, Z. Nat.forsch. A 16, 1146 (1961).

[2] R.J. Jelitto, Z. Nat.forsch. A 19, 1580 (1964).

[3] W. Haubenreisser, W. Brodkorb, A. Corciovei, G. Costache, Phys. Status Solidi 31, 245 (1969).

[4] U. Gradmann, J. Mueller, Phys. Status Solidi 27, 313 (1968); Z. Angew. Math. Phys. 30, 87 (1970).

[5] J. Korecki, U. Gradmann, Phys. Rev. Lett. 55, 2491 (1985); Hyperfine Interact. 28, 931 (1986); Europhys. Lett. 2, 651 (1986).

[6] J. Korecki, M. Przybylski, U. Gradmann, J. Magn. Magn. Mater, 89, 325 (1990).

[7] M. Przybylski, J. Korecki, U. Gradmann, Appl. Phys. A 52, 33 (1991).

[8] G. Lugert, G. Bayreuther, Phys. Rev. B 38, 11068 (1988).

[9] G.T. Rado, Bull. Am. Phys. Soc. 2, 127 (1957).

[10] D.L. Mills, A.A. Maradudin, J. Phys. Chem. Solids 28, 1855 (1967).

[11] J. Mathon, S.B. Ahmad, Phys. Rev. B 37, 660 (1988).

[12] R. Świrkowicz, Physica B 167, 239 (1990).

[13] R. Świrkowicz, A. Sukiennicki, Physica B 149, 37 (1988).

[14] R. Świrkowicz, Phys. Status Solidi B 129, 641 (1985).

[15] R. Świrkowicz, Physica B 173, 284 (1991).

[16] D.T. Pierce, R.J. Celotta, J. Unguris, II.C. Siegmann, Phys. Rev. B 26, 2566 (1982).

[17] G.T. Rado, Phys. Rev. B 40, 407 (1989).

[18] C. Rau, J. Magn. Magn. Maler. 30, 141 (1982).

[19] C. Rau, H. Kuffner, J. Magn. Magn. Mater. 54-57, 767 (1986).

[20] J. Celotta, D.T. Pierce, G.C. Wang, S.D. Baber, G.P. Felcher, Phys. Rev. Lett. 43, 728 (1979).

[21] J. Voigt, X.L. Ding, R. Fink, G. Krausch, B. Luckscheiter, R. Plazer, U. Wöhrmann, G. Schatz, J. Magn. Magn. Mater. 93, 341 (1991).

[22] G. Bayreuther, Hyperfine Interact. 47, 237 (1989).

[23] H. Hasegawa, Surf. Sci. 182, 591 (1987). 